\title{
The Comparison of Thick and Thin Intermediate Wafer in Maxillary Le Fort I Osteotomies
}

\author{
Farnoush Mohammadi ${ }^{1}$, Naghmeh Bahrami ${ }^{1}{ }^{*}$, Aref Darvishi ${ }^{2}$ \\ ${ }^{1}$ Craniomaxillofacial Research Center, Oral and Maxillofacial Surgery Department, School of Dentistry, \\ Tehran University of Medical Sciences, Tehran, Iran \\ ${ }^{2}$ Craniomaxillofacial Research Center, Tehran University of Medical Sciences, Tehran, Iran \\ Email:*n-bahrami@sina.tums.ac.ir
}

How to cite this paper: Mohammadi, F., Bahrami, N. and Darvishi, A. (2018) The Comparison of Thick and Thin Intermediate Wafer in Maxillary Le Fort I Osteotomies. International Journal of Clinical Medicine, 9, 23-27.

https://doi.org/10.4236/ijcm.2018.91003

Received: November 8, 2017

Accepted: January 12, 2018

Published: January 16, 2018

Copyright $\odot 2018$ by authors and Scientific Research Publishing Inc. This work is licensed under the Creative Commons Attribution International License (CC BY 4.0).

http://creativecommons.org/licenses/by/4.0/

\begin{abstract}
Background: Osteotomy wafers were routinely used in orthognathic surgery for repositioning the mobilized maxilla to achieve the planned final occlusion. Objectives: The aim of the current study was to determine comparison of thick and thin intermediate wafer in maxillary Le Fort I osteotomies. Methods: This study was done in 9 patients who had maxillary prognathism or retrognathism abnormality. The maxillary cast was oriented using articulator after facebow transfer. Then photographic and cephalometric data was used to determine proper dental arch segments. All 9 patients had Le Fort I combined with mandibular sagittal split osteotomies. The Le Fort I surgery was done on lateral, septum and medial sinus of nasal and trigomaxillary. The cast was removed from the base articulator and think and thick wafers were fabricated for each. Then the wafers were fixed in 1, 2 and $3 \mathrm{~mm}$ anterior $\left(A_{1}, A_{2}\right.$ and $A_{3}$, respectively). After mobilization of the maxilla and adequate bone removal, the jaws were held in occlusion with the thin intermediate wafer. The maxilla was then located against the stable part of the facial skeleton above using the yet unoperated mandible as an autorotated guide. Then the superior reposition $>1$ or $<1 \mathrm{~m}$ between two wafers was determined. Results: According to the results, the superior reposition was higher in thin wafers fixed in $A_{3}>A_{2}$ compared to $A_{1}$. Also, the same result was detected in thick wafers fixed in $A_{3}>A_{2}$ compared to $A_{1}$, respectively. However, there was no significant difference in both thin and thick wafers in each fixed locations. Conclusion: These results suggest thick wafers have acceptable results in maxillary Le Fort I osteotomies.
\end{abstract}

\section{Keywords}

Thick Wafer, Thin Wafer, Le Fort I, Maxillary Osteotomy 


\section{Introduction}

Dentofacial abnormality is a condition mainly affecting jaw and dentition. This deformity has adverse effect on chew, speech impairment, unacceptable esthetics, temporomandibular joint disorders and psychological issues [1]. In severe conditions, the orthodontic treatment is not able to achieve the normal asymmetry and co-application of orthodontic treatment and maxillary inferior repositioning surgery is the best treatment option [2]. The aim of the orthognathic surgery relies on accurate repositioning of the jaws based on careful preoperative plan. Anteroposterior and lateral movements of the maxilla are readily reproduced at surgery [3].

Osteotomy wafers were routinely used in orthognathic surgery as an intermediate guide for repositioning the mobilized maxilla relative to the intact mandible to achieve the planned final occlusion and post-operative proprioceptive guidance [4]. The wafer enables the dental arches to be put in any desired pre-planned position as well as eliminates intra-operative decisions [4]. Additionally, the wafer can be used to stabilize the dental arch segments in the planned position [5]. Two types of the wafers were produced, thin and thick. The thick wafer was fabricated before autorotation of the mandibular cast and the thin after the autorotation [4]. The wafer facilitates the dental arches to be put in desired prepared position [6]

Based in the literature, the application of the thinnest wafers was conceptually preferred than the thick ones by the surgeons [6]. In another study, Bamber and Harris (1995) reported no difference found between the thick and the thin wafers in 74 percent of the patients [7]. Segmental Le Fort I maxillary osteotomy is a beneficial technique in the management of transverse and vertical maxillary discrepancies [8] as well as the correction of malocclusion and maxillomandibular deformities [9]. Also, Le Fort I allows for movement in all three planes [9]. Since no studies, to the best of our knowledge, have been conducted on role of thick than thin intermediate wafer in maxillary Le Fort I osteotomies. So, the aim of the current study was to determine comparison of thick and thin intermediate wafer in maxillary Le Fort I osteotomies.

\section{Material and Methods}

This study was done in 9 patients in one year, who had maxillary prognathism or retrognathism abnormality. The inclusion criteria were patients who had maxillary prognathism or retrognathism abnormality, not being previous ortrognathism surgery, asymmetry in upper maxilla. The volunteer patients who had inclusion criteria informed about the study and signed the form. The maxillary cast was oriented usingarticulator after facebow transfer. Then photographic and cephalometric data was used to determine proper dental arch segments. All 9 patients had Le Fort I combined with mandibular sagittal split osteotomies. The cast was removed from the base articulator and think and thick wafers were fabricated for each. Then the wafers were fixed in 1,2 and $3 \mathrm{~mm}$ anterior $\left(A_{1}, A_{2}\right.$ and $\mathrm{A}_{3}$, respectively). 


\section{Model Surgery}

Reference lines were drawn in order to make all movements visible in a three dimension. Horizontal reference line was drawn at $5 \mathrm{~mm}$ above the articulating ring and parallel to the occlusal plane. The Le Fort I surgery were done on lateral, septum and medial sinus of nasal and trigomaxillary. Then upper maxilla fixed in proper coordination. After mobilization of the maxilla and adequate bone removal, the jaws were held in occlusion with the thin intermediate wafer. The maxilla was then located against the stable part of the facial skeleton above using the yet unoperated mandible as an autorotated guide. Then the superior reposition $>1$ or $<1 \mathrm{~mm}$ between two wafers were determined (Figure 1).

\section{Results}

The demographic information of the patients included into study is shown in Table 1. Nine patients included into the study ( 4 men and 5 women) with the mean age of 22.5 years.

According to the results, the superior reposition was higher in thin wafers fixed in $A_{3}>A_{2}$ compared to $A_{1}$. Also, the same results was detected in thick wafers fixed in $A_{3}>A_{2}$ compared to $A_{1}$, respectively. However, there was no significant difference in both thin and thick wafers in each fixed locations (pvalue $\leq$ 0001) (Figure 2).

\section{Discussion}

As observed in this study, there was no significant difference in both thin and thick wafers in each fixed locations. Based on the data, thick wafers have acceptable results in maxillary Le Fort I osteotomies. Recently, Firouzei in a comparative study of thin and thick surgical splint during Le Fort I osteotomy in vertical maxillary excess reported the use of thick splints will cause the maxilla to take a more correct position [10]. Also, Bamber and Harris reported no difference found between the thick and the thin wafers in 74 percent of the patients [7]. It

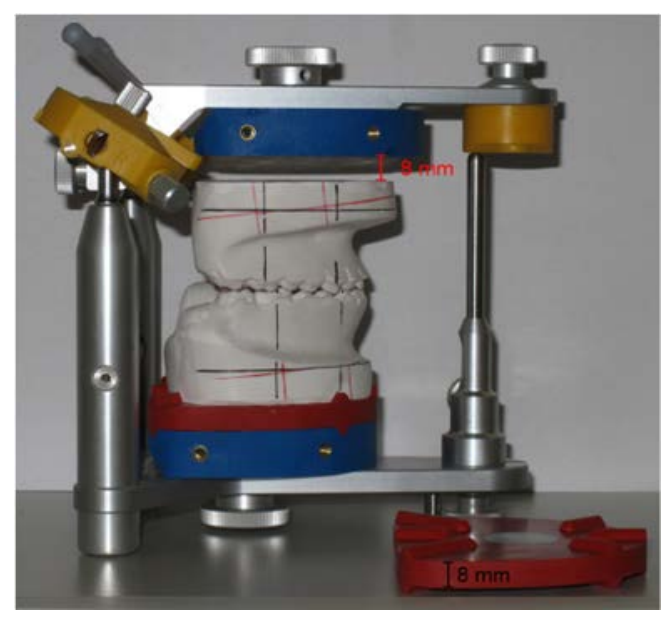

Figure 1. Acrylic device for fabrication of thick and thin wafers. 


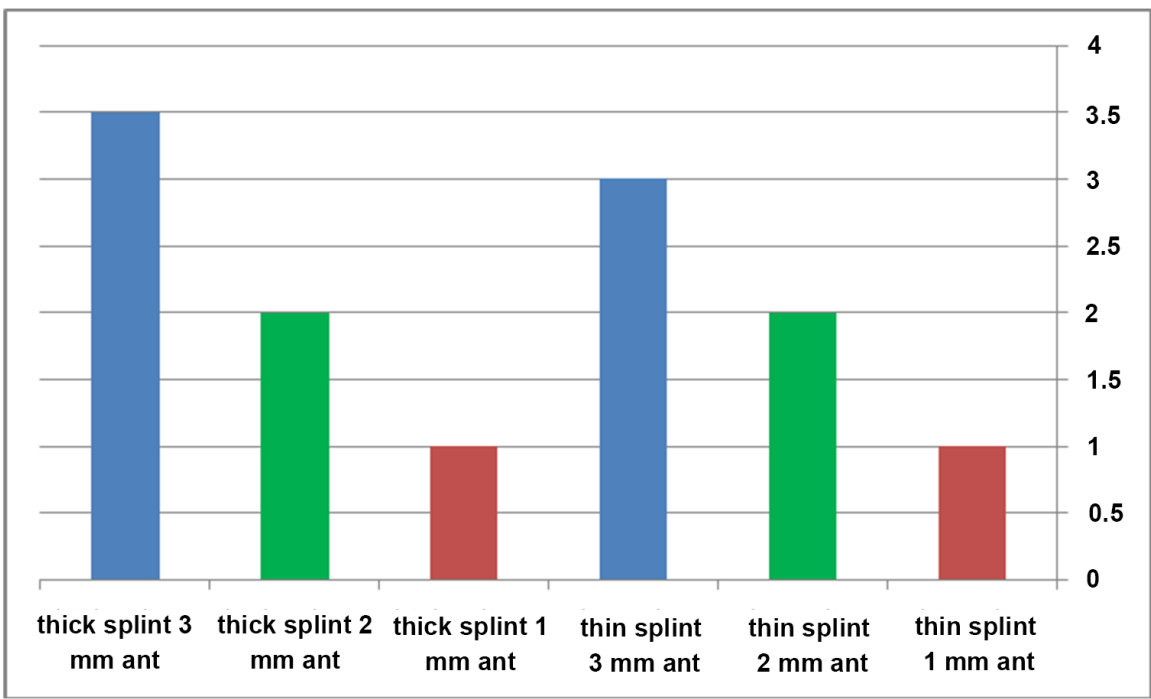

Figure 2. The results of the application thin and thick wafer on superior reposition.

Table 1. The demographic information of the patients included into study.

\begin{tabular}{cccc}
\hline Sex & & Frequency (\%) & \\
male & $4(44.5 \%)$ & & \\
female & $5(55.6 \%)$ & & Maximum \\
Age & Mean \pm Std. & minimum & 27.00 \\
\hline
\end{tabular}

has also been suggested thicker wafer posteriorly $(<2 \mathrm{~mm})$ provides some room for upward recoiling of the condyle postoperatively [11]. As showed, the surgeons don't like thick wafers and the thin wafer is much more preferred [12] [13].

Thick wafer proved to be more revealing of posterior bone interference on autorotating the mandibular and maxillary block for plate fixation [7]. There is a belief thick wafer has wrong position or manipulate, so because of that operator's prefer thin wafers [7]. Similar to thin wafer, the thick wafer was not influenced by the articulator's autorotation of the mandibular cast [7]. In our study the final discrepancy and positioning of the osteotomized mandible was similar between 2 types of the wafers which was in agreement with previous reports [12]. The thick wafer eliminated simulation of the mandibular autorotation [1].

In our study, thick wafers have acceptable results in maxillary Le Fort I osteotomies. The LeFort I osteotomy is commonly used for the correction of malocclusion, maxillomandibular deformities and dentofacial asymmetries [9]. However, there was no previous paper on effect of thin or thick wafers in maxillary Le Fort I osteotomies. So, we were not able to compare our results with it. In conclusion these results suggest thick wafers have acceptable results in maxillary Le Fort I osteotomies. Of the limitations of the current study was to limit sample size of the patients. We think further researches needed to determine the accuracy of the results. 


\section{Acknowledgements}

The authors thank the craniomaxillofacial research center, Tehran University of Medical Sciences.

\section{Conflict of Interest}

None declared.

\section{References}

[1] Uniyal, S., Chhag, S., Anilkumar, S., Jayakumar, N. and Patel, M. (2015) A Systematic Approach for Splint Fabrication in Orthognathic Surgery: Case Series. IJSS Case Reports \& Reviews, 2, 5-9.

[2] Gordon, J.M., Rosenblatt, M., Witmans, M., Carey, J.P., Heo, G. and Major, P.W. (2009) Rapid Palatal Expansion Effects on Nasal Airway Dimensions as Measured by Acoustic Rhinometry. A Systematic Review. The Angle Orthodontist, 79, 1000-1007. https://doi.org/10.2319/082108-441.1

[3] Convens, J.M.C., Kiekens, R.M.A., Kuijpers-Jagtman, A.M. and Fudale, P.S. (2015) Stability of Le Fort I Maxillary Inferior Repositioning Surgery with Rigid Internal Fixation: A Systematic Review. International Journal of Oral and Maxillofacial Surgery, 44, 609-614. https://doi.org/10.1016/j.ijom.2014.12.015

[4] Kretschmer, W.B., Baciut, G., Baciut, M., Zoder, W. and Wangerin, K. (2010) Stability of Le Fort I Osteotomy in Bimaxillary Osteotomies: Single-Piece versus 3-Piece Maxilla. Journal of Oral and Maxillofacial Surgery, 68, 372-380. https://doi.org/10.1016/j.joms.2009.09.053

[5] Arpornmaeklong, P., Heggie, A.A. and Shand, J.M. (2003) A Comparison of the Stability of Single-Piece and Segmental Le Fort I Maxillary Advancements. Journal of Craniofacial Surgery, 14, 3-9. https://doi.org/10.1097/00001665-200301000-00002

[6] Bamber, M.A. and Vachiramon, A. (2005) Surgical Wafers: A Comparative Study. The Journal of Contemporary Dental Practice, 6, 99-106.

[7] Bamber, M.A., Abang, Z., Harris, M., et al. (1999) The Effect of Posture and Anaesthesia on Occlusal Relationship in Orthognathic Surgery. Journal of Oral and Maxillofacial Surgery, 57, 1164-1172. https://doi.org/10.1016/S0278-2391(99)90476-7

[8] Ho, M.W., Boyle, M.A., Cooper, J.C., Dodd, M.D. and Richardson, D. (2011) Surgical Complications of Segmental Le Fort I Osteotomy. British Journal of Oral and Maxillofacial Surgery, 49, 562-566. https://doi.org/10.1016/j.bjoms.2010.09.018

[9] Buchanan, E.P. and Hyman, C.H. (2013) LeFort I Osteotomy. Seminars in Plastic Surgery, 27, 149-154. https://doi.org/10.1055/s-0033-1357112

[10] Firouzei, G., Rahimi, A., Tabrizi, R. and Pouzesh, A. (2013) The Comparative Study of Thin and Thick Surgical Splint during Le Fort I Osteotomy in Vertical Maxillary Excess. Journal of Craniofacial Surgery, 24, e279-e283. https://doi.org/10.1097/SCS.0b013e31828f2a3c

[11] Bamber, M.A. and Harris, M. (1995) The Role of the Occlusal Wafers in Orthognathic Surgery: A Comparison of Thick and Thin Intermediate Osteotomy Wafers. Journal of Cranio-Maxillofacial Surgery, 23, 369-400. https://doi.org/10.1016/S1010-5182(05)80137-4

[12] Harris, M. and Reynolds, I.R. (1991) Fundamentals of Orthognathic Surgery. W B Saunders Company, London, 8-85

[13] Proffit, W.R. and White, R.P. (1991) Surgical-Orthodontic Treatment. Mosby-Year Book, St. Louis. 